\title{
RELATIONSHIP BETWEEN WEIGHT AND SLEEP ARCHITECTURE IN CHILDREN
}

\author{
J.F. Chocano ${ }^{1}$, C.S. Sendon ${ }^{2}$
}

${ }^{1}$ Pediatric Pulmonology, Children Hospital of Kings Daughters, Eastern Virginia Medical School, ${ }^{2}$ Pediatrics-Sleep Medicine, Children Hospital of Kings Daughters, Norfolk, VA, USA

Rationale: Overweight has been associated with sleep problems in children. It is important to identify if weight gain change the sleep architecture.

Objective: Evaluate overweight as a risk factor for sleep architecture disturbances.

Methods: This is a retrospective review of 224 polysomnograms (PSG) performed between January and December 2006 in a population between 2 and 20 years old. The patients were classified in three groups: normal weight, overweight and obese, underweight subjects were excluded; Total Sleep Time, Sleep Efficiency, Sleep Latency, REM duration, slow wave duration (stage 3), light sleep duration (sleep 1 and stage 2), daytime sleepiness, arousal index, age, gender, ethnicity, height, weight, BMI, and the Apnea Hypopnea Index (AHI) were evaluated.

Results: 123 of patient were male and 101 female, 120 were Caucasians and 92 African American; 100 patients had normal weight, 26 patients were overweight and 98 patients were obese. No significant relationship was found between weight and Apnea-Hypopnea index ( 0.266 Kruskal Wallis test). The normal weight group showed normal sleep stages durations and normal sleep latency. Overweight group showed more stage 3 duration, more REM duration and a tendency to decreased sleep latency. The obese group showed more severe OSAS, decrease sleep latency, decrease REM duration, decrease stage 3 duration and increase daytime sleepiness.

Conclusion: Maintaining a normal weight helps to conserve normal sleep architecture. Overweight produce an increment of deep sleep and decreased sleep latency, which are the earliest changes noticed in the sleep architecture. Obesity produce a significant disturbance in the sleep architecture. 